\title{
Wideband RF Radiation from a Nonlinear Transmission Line with a Pre-magnetized Ferromagnetic Core
}

\author{
J.-W. Ahn ${ }^{1}$, S. Y. Karelin ${ }^{2}$, V. B. Krasovitsky ${ }^{2}$,H.-O. Kwon ${ }^{1 *}$, I. I. Magda ${ }^{2}$, \\ V. S. Mukhin'2, O. G. Melezhik², and V. G. Sinitsin ${ }^{2}$ \\ ${ }^{1}$ Hanwha Corp., Gumi, Kyungbuk 39370, Korea \\ ${ }^{2}$ National Science Center 'Kharkov Institute of Physics and Technology', Kharkov 61108, Ukraine
}

(Received 8 March 2016, Received in final form 27 June 2016, Accepted 1 July 2016)

\begin{abstract}
Experimental results and data of numerical simulations are presented, concerning generation of wideband radio frequency (RF) oscillations in a nonlinear transmission line (NLTL) which contains a pre-magnetized core of ferrite material. Emphasis is made on the means for extracting the RF signal from the line, in order to radiate it into free space. Antennas of two types that can be used for the purpose are considered, both featuring a coaxial design. This permits availing of the principal advantages of coaxial systems, such as operation in the mode of a traveling TEM wave; wide range of the transmitted frequencies, and a reasonably simple design. The antennas studied, specifically a disc-cone dipole (DCD) and an impulse reflector antenna ('Half-IRA', or HIRA type) differ significantly in effective width of the radiated spectrum and in spatial characteristics of the radiated field in far region.
\end{abstract}

Keywords : quasi-harmonic wideband signal, pulsed current-excited NLTL, disc-cone dipole, HIRA

\section{Introduction}

The possibility of direct conversion of a high-voltage 'video' pulse into a radio frequency signal in devices that employ excitation of magnetic moment precession in a ferrite-filled nonlinear transmission line (NLTL) is of great importance for the high-power microwave technology [1-3]. The uniqueness of such systems stems from the wide range of frequency tuning in the generated signal (that may span from hundreds of $\mathrm{MHz}$ to a few $\mathrm{GHz}$ ), which is controlled only by the injected shock pulse of electric current and the external magnetic field. Recent experiments show that the output power of repeated quasiharmonic wideband (QHWB) RF signals can exceed hundreds of megawatts [4]. Obviously, the radiating antennas of such systems must possess a number of characteristics to distinguish them from traditional narrowband radiators. To be able to canalize and radiate a specific QHWB RF signal, like a damped sinusoid, they should present a sufficiently broad passband, providing for minimum energy losses over the range of frequencies

CThe Korean Magnetics Society. All rights reserved.

*Corresponding author: Tel: $+82-54-467-8784$

Fax:+82-54-467-8780, e-mail: waveguy5@hanwha.com generated by the NLTL. It is also important to note that due to the relatively short duration of the emitted signal (about several nanoseconds) these radiating antennas should demonstrate the properties of impulse antennas operating in the traveling wave mode. Some of essential design features of such antennas are dictated by the geometry and topology of the associated transmission lines, the NLTL in particular. The antennas with a coaxial input port are fed by a coaxial waveguide of relatively large cross section, providing the necessary electric strength of the structure. For example, if the pulsed current signal through the NLTL is $I=U_{0} / Z_{\mathrm{NL}}=1 \mathrm{kA}$ and the line impedance in the presence of a saturated ferrite makes $Z_{\mathrm{NL}} \approx 50 \Omega$, then with an amplitude modulation ratio at the output from the NLTL being $k_{\mathrm{MU}}=0.3-0.4$, the power level $P_{\mathrm{OSC}}$ of the RF oscillations, $P_{\mathrm{OSC}} \approx 1 / 2\left(k_{\mathrm{MU}}\right.$ $\left.I_{0}\right)^{2} Z_{\mathrm{NL}}$, can be as high as 2-4 MW. Accordingly, the electric field strength at the ferrite-dielectric interface can approach to the critical value of $\sim 100 \mathrm{kV} / \mathrm{cm}$.

Despite the impressive history of NLTL studies [1-7], experiments on extracting and radiating the intense RF signals have been detailed in just a few papers, e.g. $[4,5]$. In some of these the output signal from a coaxial NLTL was fed to a circular waveguide through a TEM-to-TE 11 mode converter and further on to a conical horn antenna. 
The converter, although involving an adjustable low pass filter, leaves the structure a frequency-dependent or, rather a narrow band device, owing to the presence of the hollow waveguide whose dispersion properties are determined by its radius. Due to this design the pulsed waveform in the antenna acquires a specific shape with a slow increase of the envelope and a decrease over several RF periods, finally expanding the RF signal's duration time.

In this paper we present our results on generation of QHWB radio frequency oscillations in NLTLs with a premagnetized ferromagnetic core [6,7], and of radiation experiments with two types of moderately wideband impulse antennas.

\section{RF Oscillations in the Ferrite-filled Non-linear Line}

Figure 1 presents a schematic of the QHWB pulse generator exercising induction of damped sinusoidal $\mathrm{RF}$ oscillations in the ferrite core of a NLTL, together with the antennas for radiating and receiving the microwaves. The high-voltage pulse generator (output impedance $Z_{\text {out }}$ $=37 \Omega$ ) based on a Tesla transformer, formed voltage pulses of a 8 ns width (at half-maximum level) and 50$150 \mathrm{kV}$ amplitude. The transmission system consisted of two short $(0.2 \mathrm{~m})$ coaxial transmission lines, TL1 and TL2 with respective impedances $Z=37 \Omega$ and $Z=50 \Omega$, and the coaxial NLTL placed between them. The NLTL was filled with ferrite beads of sizes $D_{2} \times D_{1} \times W=20$ $\mathrm{mm} \times 12 \mathrm{~mm} \times 6 \mathrm{~mm}\left(\mathrm{D}_{1}\right.$ is the inner diameter; $D_{2}$ the outer diameter, and $\mathrm{W}$ the bead length). The ferrite grade was 200VNP (permeability $\mu=200$ ), and the total length of the ferrite material made $0.85 \mathrm{~m}$. The inner diameter $\mathrm{D}_{3}$ of the outer conductor in the coaxial sections was equal to $26 \mathrm{~mm}$. The size of the central conductor in TL1, TL2 and NLTL was chosen from between $8 \mathrm{~mm}$ and 12 $\mathrm{mm}$, depending on the matching conditions required. The high-voltage insulation in all sections of the coaxial system was provided by means of transformer oil (dielectric constant equal to 2.25). The bias magnetic field was created by a $1 \mathrm{~m}$ long solenoid, wound directly on the housing of the NLTL. The signals at the NLTL input and output were measured with capacitive dividers located at the center of the short coaxial transmission sections. The transmitting antennas could be connected directly to the output coaxial section.

The experimental parameters are shown in Table 1. The results obtained for a coaxial NLTL $\left(D_{3} / D_{1}=26 \mathrm{~mm} / 12\right.$ $\mathrm{mm})$ filled with the 200VNP-type NiZn ferrite $\left(D_{2} / D_{1}=\right.$ $20 \mathrm{~mm} / 12 \mathrm{~mm}$; magnetic saturation level $H_{0}=96 \mathrm{kA} / \mathrm{m}$ ) were as follows $[6,7]$. The carrier-free voltage pulses fed

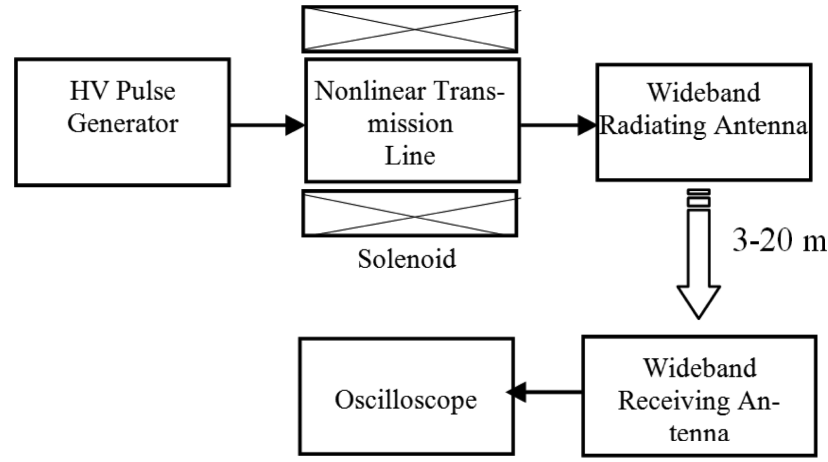

Fig. 1. Schematic of the HF radiation experiment.
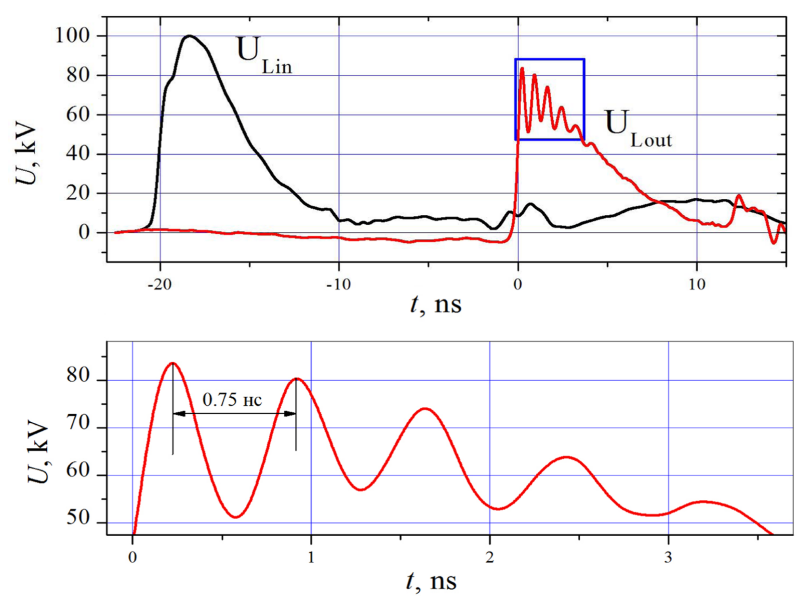

Fig. 2. (Color online) Typical voltage waveforms in the NLTL: the input (black) and output (red) voltage pulses $\left(U_{\mathrm{NLout}}=72 \mathrm{kV}, H_{0}=96 \mathrm{kA} / \mathrm{m}, f_{\mathrm{OSC}}=1.3 \mathrm{GHz}\right)$.

to the ferrite-filled NLTL to drive magnetic moment precession, varied in peak magnitude between $50 \mathrm{kV}$ and $150 \mathrm{kV}$, giving rise to output pulses with a much shorter front edge $(<0.5 \mathrm{~ns})$ and a few quasi-periods of decaying RF oscillations of peak amplitude $10 \mathrm{kV}$ to $17 \mathrm{kV}$. Depending on the magnitude of the driving voltage the oscillation spectrum that expanded from $0.6 \mathrm{GHz}$ to $>2.5$ $\mathrm{GHz}$ demonstrated noticeable discrete peaks. A driving pulse of peak magnitude $U_{L \text { in }}=100 \mathrm{kV}$ normally produced a sharp-edged output pulse of a smaller size, $U_{\text {Lout }}=72$ $\mathrm{kV}$, followed by a few periods of oscillations. This pulse can be interpreted as a composite signal involving one or a few quasi-harmonic components, plus a broad spectral background. Assuming that the line impedance approaches $50 \mathrm{Ohm}$ as the ferrite gets saturated, we can estimate the output power carried by that pulse as $P_{\text {Lout }} \approx 100 \mathrm{MW}$, and the parameters of the RF oscillations as $U_{\mathrm{OSC}}=17$ $\mathrm{kV}$, whence the RF power is $P_{\mathrm{OsC}} \approx 3 \mathrm{MW}$. Thus, the DCto-RF conversion efficiency is within $k_{\mathrm{MP}}=0.04-0.1$ over the frequency band 0.6-2.0 GHz, Fig. 2. Next, the composite RF signal is to be radiated, with minimum reflection 
Table 1. Typical characteristics of the experiments with NLTL and different radiating antennas.

\begin{tabular}{cccc}
\hline \hline HV pulse generator & NLTL & Radiating antennas & Receiving antennas \\
\hline$t_{\mathrm{P}}=8-14 \mathrm{~ns}$, & $t_{\mathrm{P}}=8 \mathrm{~ns}, f=0.6-2.0 \mathrm{GHz}$, & TX1: DCD & 1. Strip-line horn (WB) \\
$U=50-150 \mathrm{kV}$ & $I \approx 3 \mathrm{kA}, Z_{\mathrm{NLsat}} \approx 50 \mathrm{Ohm}, k_{\mathrm{MU}}=0.2-0.3$ & TX2: HIRA & 2. Standard horn $0.8-12 \mathrm{GHz}$ (WB) \\
\hline
\end{tabular}

losses, via a matched antenna into free space.

\subsection{Choice of a WB antenna for the coaxial feedline}

The topology of the principal element in the RF pulse generator, namely the coaxial NLTL, determined the choice of both the design and characteristics of the antennas which were

(a) a conical antenna with a disc-shaped ground plane (disc-cone dipole, DCD), and

(b) a half-circular reflector impulse antenna ('Half-IRA' type, [8]) in which the parabolic reflector is driven by a symmetrical dual strip-line horn. When selecting these as QHWB emitters, the following advantages of coaxial systems are taken into account,

- simplicity of design, allowing easy matching of the antenna input to the NLTL's coaxial output;

- operation in the mode of a traveling TEM wave, hence with a wide range of transmitted frequencies;

- specialty of the pulse response peculiar to TEM guiding systems which brings forth only small distortions of the radiated RF signal, thus determining the broadband quality of the antennas within the frequency range of interest;

- possibility to form radiation patterns with a variety of directive gain factors and a relatively high value of the peak gain (in the case of a HIRA).

In experiment, the radiated power is detected and quantified with the aid of a receiving antenna and a broad-band measuring instrument (e.g., digital oscilloscope). We have used two receiving antennas (see Table 1) that can be described as wideband (WB). Using a calibrated receive antenna one might be able to estimate the radiated power in the far-field region and compare the relative efficiency of the transmitting antennas. The respective estimates have been done in this paper for transmitting antennas of both types through numerical simulation and with the use of real measured data.

The RF power $P$ at the input to a receive antenna can be written as $P(\mathbf{r})=A S(\mathbf{r})$, where $A$ is the effective collecting area; $\mathbf{r}$ the position vector of the antenna (or of a point within its aperture plane); $\mathbf{E}(\mathbf{r}, t)$ and $\mathbf{H}(\mathbf{r}, t)$ the RF electric and magnetic field vectors, respectively, and $\boldsymbol{S}(\mathbf{r}, t)=[\mathbf{E}(\mathbf{r}, t) \times \mathbf{H}(\mathbf{r}, t)]$ the Poynting vector. The average power arriving over time interval $T$ (centered at $\left.t_{0}\right)$ is

$$
<P>=A<S>\text {, where }\left\langle\mathbf{S}(\mathbf{r})>=(1 / T) \int_{t_{0-T} / 2}^{t_{0}+T} d t \mathbf{S}(\mathbf{r}, t)\right. \text {. Sub- }
$$

stituting Fourier representations for $\mathbf{E}(\mathbf{r}, t)$ and $\mathbf{H}(\mathbf{r}, t)$, and resorting to Maxwell equations to express the magnetic vector $\mathbf{H}(\omega, \mathbf{k})$ via $\mathbf{E}(\omega, \mathbf{k})$, we have

$$
\begin{aligned}
<\boldsymbol{S}(\mathbf{r})>= & \frac{1}{\mu_{0} \mathrm{~T}} \iint \mathrm{dk}_{1} \mathrm{dk}_{2}\left(1 / \omega_{2}\right)\left[\mathbf{E}\left(\mathrm{k}_{1}\right) \times\left[\mathbf{k}_{2} \times \mathbf{E}^{*}\left(\mathrm{k}_{2}\right)\right]\right] \\
& \exp \left(\mathrm{i}\left(\omega_{1}-\omega_{2}\right) \mathrm{t}_{0}+\operatorname{ir}\left(\mathbf{k}_{1}-\mathbf{k}_{2}\right)\right) \frac{\sin \left(\omega_{1}-\omega_{2}\right) \mathrm{T} / 2}{\left(\omega_{1}-\omega_{2}\right) 1 / 2} .
\end{aligned}
$$

Here $d k=d \omega d \mathbf{k} ; \mathbf{E}(k)=\mathbf{E}(\omega, \mathbf{k})$ is the spatial and frequency Fourier component of the field vector $\mathbf{E}(\mathbf{r}, t)$; $\mathbf{k}$ the corresponding wave vector (the asterisk $^{*}$ denotes complex conjugation), and $\mu_{0}$ is the permeability of free space. Apparently, Eq. (1) represents the total power (per unit area) that potentially can be acquired by the receive antenna, including contributions from all directions of field incidence and all frequencies of the radiated spectrum. In fact, the receive antenna accepts just a part of this total power, in accordance with its frequency selectivity and spatial directional properties. The effective collecting area $A$ is different from the mechanical area of the antenna's aperture, being determined by the reception pattern which is a frequency dependent function. In their turn, the radiated spectral field components $\mathbf{E}(\omega, \mathbf{k})$ in Eq. (1) are fully determined by directional properties of the transmit antenna and frequency composition of the signal to drive that [9]. We have come to the necessity of a detailed study of directional properties of the three antennas used in the radiation experiment.

\subsection{Spectral and directional properties of the DCD and HIRA WB antennas, and the strip-line receiving horn}

The notions of antenna pattern and directive gain which are traditional in the theory of narrow band antennas remain applicable in the wideband case too, but only to separate spectral components of the composite transient signals. A 'universal' time-domain parameter that may be used to characterize the directivity of a transmitting antenna with respect to signals of any kind (either $\mathrm{CW}$ narrow band, or broadband pulses) is the factor of merit (FOM), or relative electrodynamic potential [10]. The definition, $\mathrm{FOM}=E(\mathbf{r}) r / U_{O S C}$, can be re-written in terms of the 
magnitudes of Eq. (1) as

$$
\mathrm{FOM}=\left(c \mu_{0}<S(\mathbf{r})>\right)^{1 / 2} r / U_{O S C} .
$$

Here $E(\mathbf{r})$ is maximum far-region electric field strength at point $\mathbf{r}$ separated by distance $r$ from the source of radiation; $S(\mathbf{r})$ is power flux at that point; $c$ is the speed of light, and $U_{\mathrm{OSC}}$ the peak amplitude of RF voltage oscillations in the radiator. Monochromatic directive gains of all the participant antennas and FOMs of the wideband transmit antennas have been studied with the use of a 3D numerical simulation code, viz. the CST MW Studio Suite
[11]. The results are presented in Figs. 3-5.

The design elements of the antennas are shown in Figs. 3(a), 4(a) and 5(a). Both transmit systems have a short (approx. $0.2 \mathrm{~m}$ ) TEM feedline and a 'ground plane' electrode. For matching the antennas to the NLTL at the low frequency end, a distributed absorbing load is used, implemented as a set of low-inductance resistors with a total active resistance corresponding to the NLTL's impedance as predicted for the nearly saturated magnetization state of the ferrite $\left(Z_{\mathrm{NLsat}} \approx 50 \Omega\right)$.

The test signals used in the simulation were the experi-

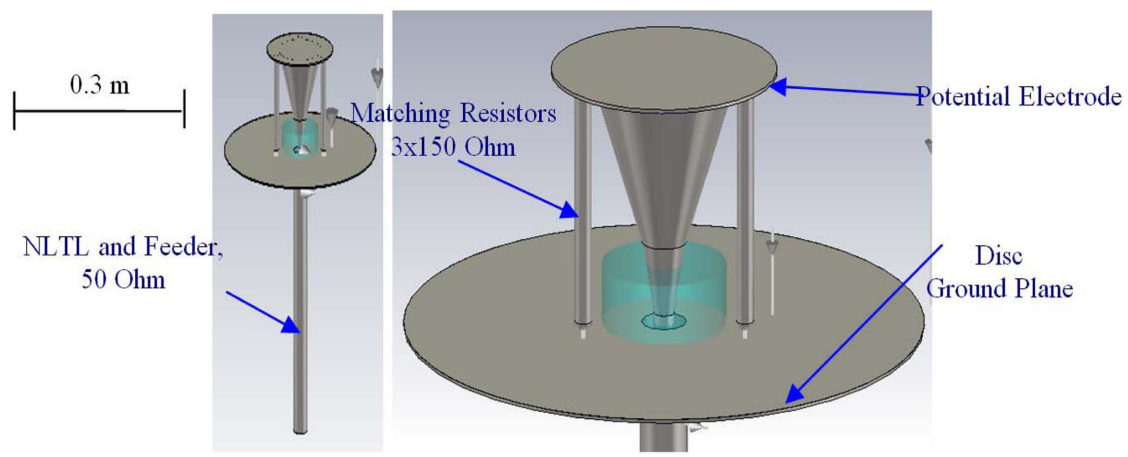

(a) Geometry of radiating antenna TX1 (DCD)
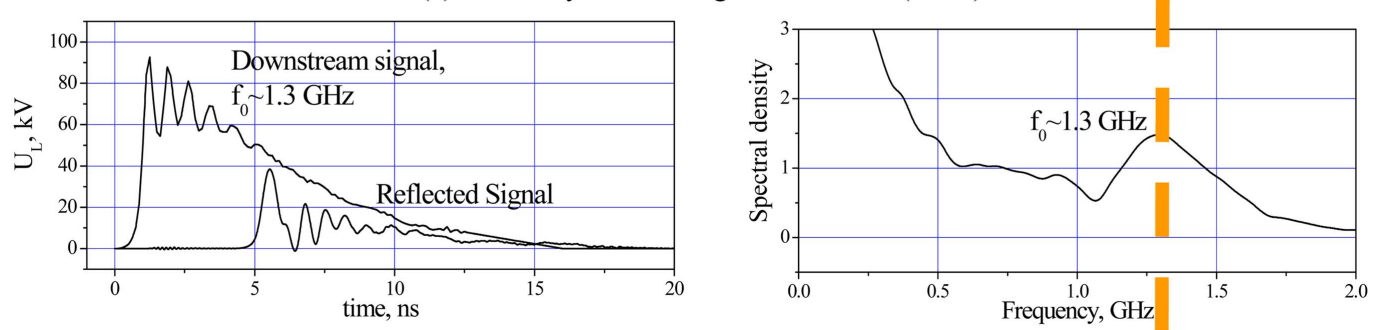

(b) Downstream and reflected signal in the feedline and radiated power spectrum
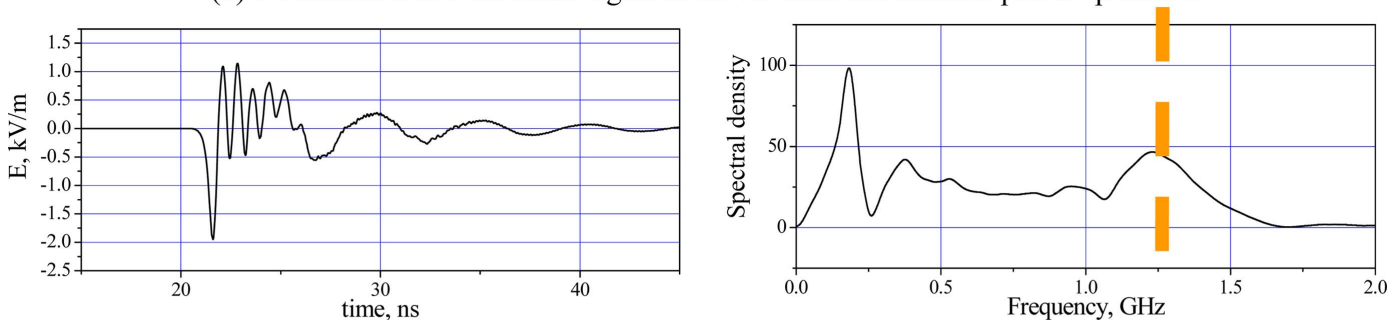

(c) Pulsed field strength, $\mathrm{E}$ and its spectrum in the far field zone $(\mathrm{L}=6 \mathrm{~m})$
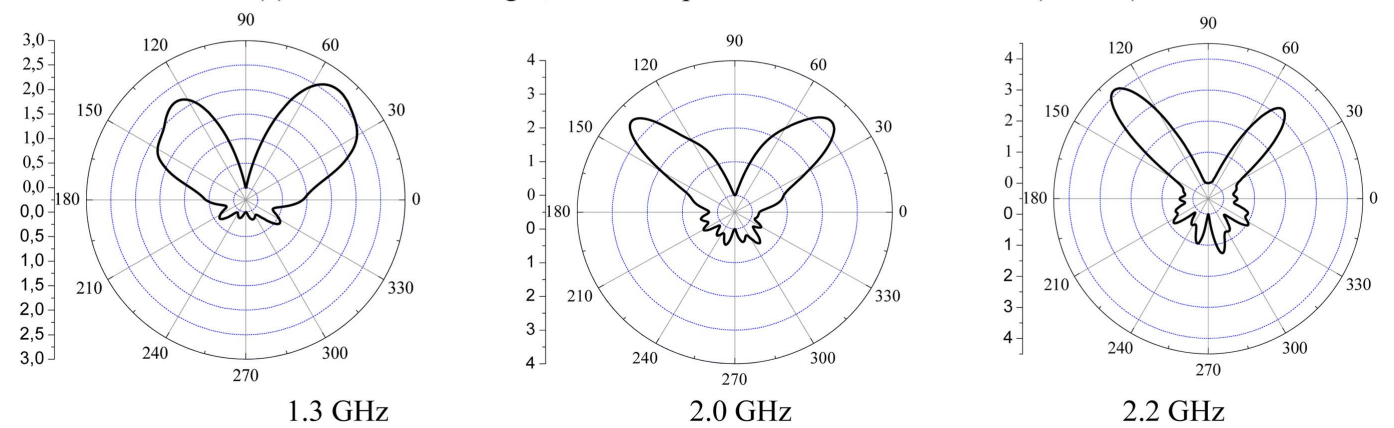

$2.2 \mathrm{GHz}$

(d) Vertical-plane sections of power patterns of the DCD antenna at different frequencies (linear scale, a.u.)

Fig. 3. (Color online) Simulation results for the disc-cone dipole antenna (Antenna TX1). 


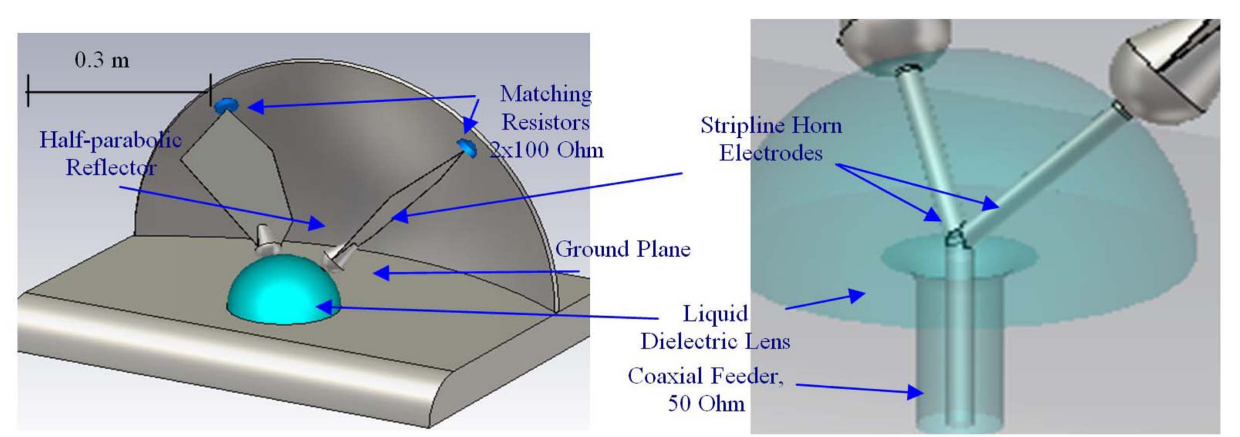

(a) Geometry of radiating antenna TX2 (HIRA)
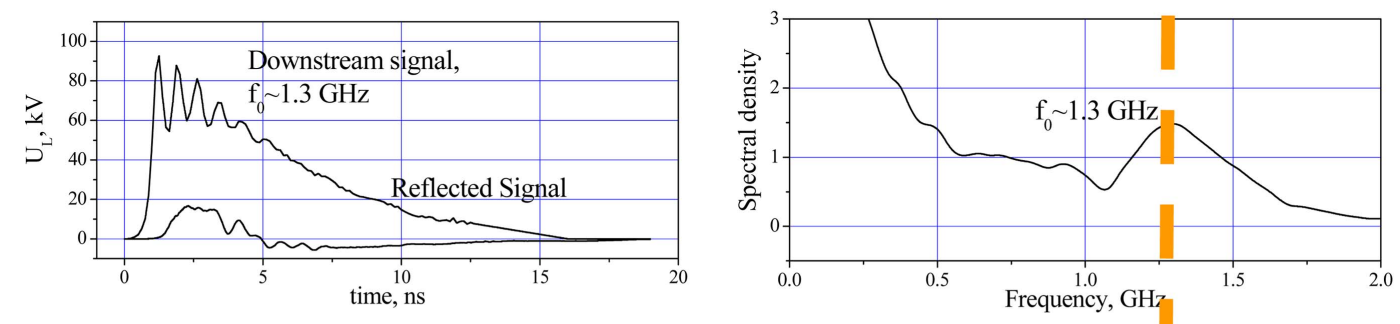

(b) Downstream and reflected signal in the feedline, and spectrum of the radiated power
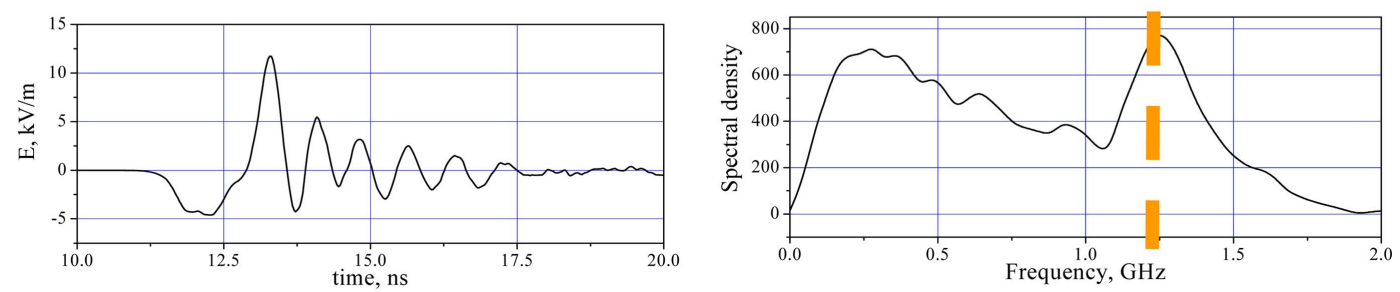

(c) Pulsed field strength, $\mathrm{E}$ and its spectrum in the far field zone $(\mathrm{L}=6 \mathrm{~m})$

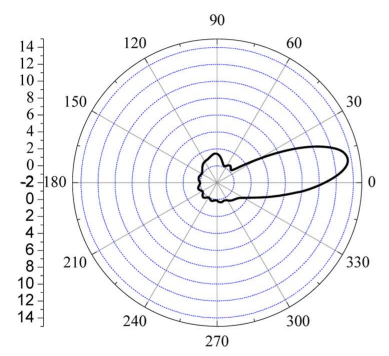

$1.3 \mathrm{GHz}$

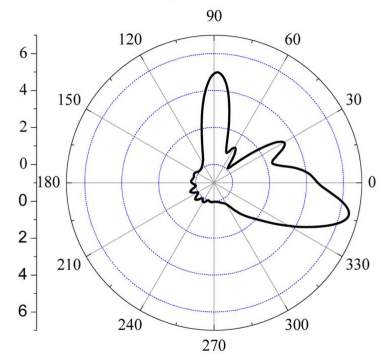

$2.0 \mathrm{GHz}$

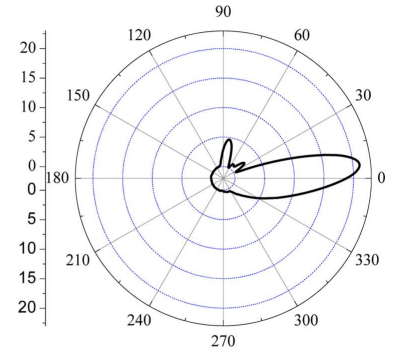

$2.2 \mathrm{GHz}$

(d) HIRA radiation patterns at different frequencies

Fig. 4. (Color online) Simulation results for the Half-Reflector Impulse Antenna.

mentally observed pulses that appeared at the NLTL output under typical conditions (see Table 1). Figure 3(b) and Fig. 4(b) show the calculated waveforms traveling through the coaxial feedline toward the antenna input and back, upon reflection from the antenna, as well as the spectra of these signals. The radiated signals, as shaped in the far region, and their spectra are shown in Fig. 3(c) and Fig. 4(c). As can be seen, the far-field signals demonstrate a broadband spectrum in the range of $0.2-2.0 \mathrm{GHz}$, with a pronounced peak near $1.3 \mathrm{GHz}$. The lower frequency part of this spectrum owes to the spectral content of the initial 'video' pulse. It has not been greatly changed while traveling through the ferrite but somewhat filtered in the antenna. Meanwhile, the enhanced spectral density around $1.3 \mathrm{GHz}$ is a result of QHWB generation in the NLTL.

The DCD shows a nearly uniform directivity in the azimuthal plane and a maximum gain of $1.4 \div 1.6$ at $f=$ $0.6 \div 0.9 \mathrm{GHz}$ and $1.8 \div 2.7$ at $f=1.2 \div 2.2 \mathrm{GHz}$ in the plane of the elevation-angle $\vartheta$. The angular position of this smooth maximum varies with frequency in the range of $\vartheta=30 \div 50^{\circ}$. It contrast to this, the HIRA whose reflector is driven in a frequency-independent way, practically does 
not change the direction of its maximum and demonstrates a higher gain (viz. $6 \div 18$ ) over the frequency range in question.

As can be seen from the above data, both transmit antennas can be used for radiating the QHWB pulse signals which are formed in the NLTL, with the RF component lying at $0.6 \div 2.5 \mathrm{GHz}$. To compare the data of the numerical and the real-life experiment, a UWB receiving antenna of a strip-line horn (SLH) type was used (see Fig. 5), which was placed in the far region, at a distance of $6 \mathrm{~m}$.

(The familiar estimate for the guaranteed far-field length is $L_{\mathrm{FF}}=2 D^{2} / \lambda$ where $D$ is a characteristic size of the antenna or its reflector and $\lambda$ the shortest wavelength from the spectral content of the radiated pulse). The SLH possesses a rather sharply directed pattern in the azimuthal plane with an almost identical gain of 14-30 at all the frequencies of interest (similar as an almost fixed angular position of the vertical-plane maximum, specifically $\vartheta=30^{\circ}-47^{\circ}$ ). Vertical-plane reception patterns of the SLH for $f_{0}=1.3 \mathrm{GHZ}, f_{0}=2.0 \mathrm{GHz}$ and $f_{0}=2.2 \mathrm{GHz}$ are given in Fig. 5(b).

The results of the numerical analysis described, involving the two radiating antennas and the SLH receiving antenna, are shown in Figs. 5(c) and 5(d), and summarized in Table 2. As can be seen, the simulations for the signals that could be received by the SLH have approximately the same shape for both transmit antennas, which confirms the possibility of registering wideband features of the QHWB signals generated in the ferromagnetic NLTL. The quality of antenna matching to the coaxial feed is rather different in the two cases, whereas the receiving horn demonstrates a nearly perfect matching (see column 3 of the Table). Because of different gain factors of the two radiating antennas (at all specific frequencies), the far-region field strengths produced by the DCD and the HIRA antennas (column 6 of the Table) and, accordingly, the responses of the SLH antenna are

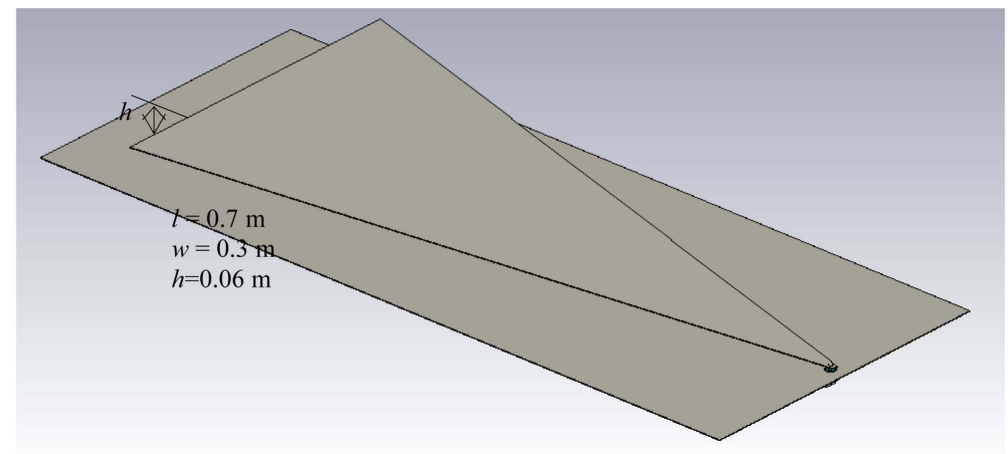

(a) General view of the SLH receive antenna

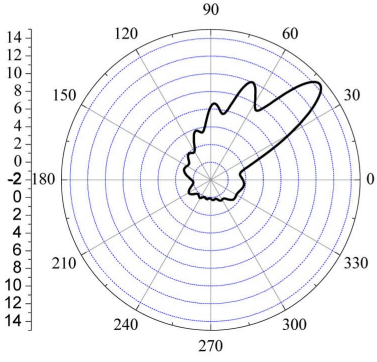

$1.3 \mathrm{GHz}$

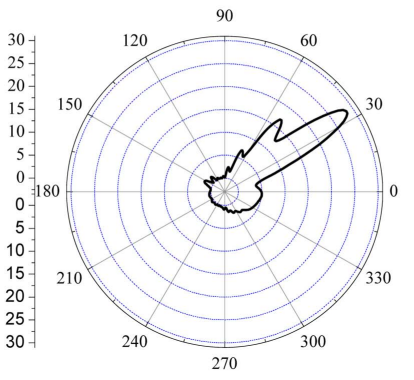

$2.0 \mathrm{GHz}$

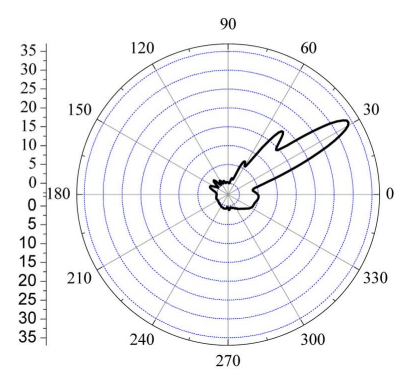

$2.2 \mathrm{GHz}$

(b) Vertical-plane reception patterns of the SLH for $\mathrm{f}_{0}=1.3 ; 2.0$, and $2.2 \mathrm{GHz}$
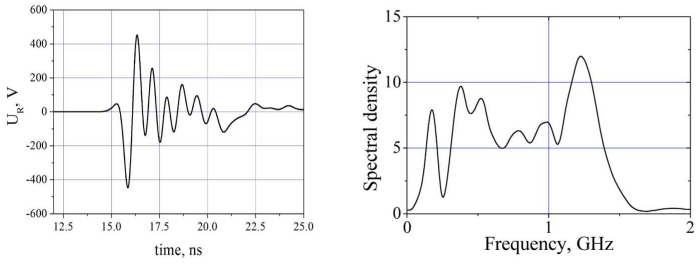

(c)
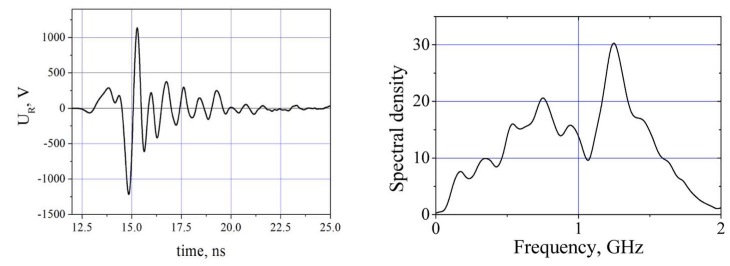

(d)

Fig. 5. (Color online) Performance of the SLH receiving antenna. RF voltage waveforms at the $50 \Omega$ output load and the corresponding spectra: (c) signals from TX1 (DCD) and (d) from TX2 (HIRA), $6 \mathrm{~m}$ away from the transmit antennas $\left(U_{\mathrm{OSC}}=17 \mathrm{kV}\right)$. 
Table 2. Computed performance parameters of the two transmit antennas and the receiving strip-line horn.

\begin{tabular}{|c|c|c|c|c|c|c|c|c|}
\hline Antenna & $\begin{array}{l}\text { Impedance, } \\
\text { Ohm }\end{array}$ & $\begin{array}{c}\text { Voltage reflection } \\
\text { factor in feeder } \\
k_{\mathrm{MU}}=U_{\text {refl }} / U_{0}\end{array}$ & $\begin{array}{c}\text { Power transfer } \\
\text { ratio, } \\
P_{R A D} / P_{O S C}\end{array}$ & $\begin{array}{c}\text { Radiated power, } \\
P_{R A D}, \mathrm{MW} \\
\left(P_{O S C}=3 \mathrm{MW}\right)\end{array}$ & $\begin{array}{c}\text { Field strength at } \\
\text { distance } r \\
E(r) \mathrm{kV} / \mathrm{m}\end{array}$ & FOM & Max. gain & $\begin{array}{c}\text { Directive gain } \\
\text { for } \\
\vartheta=0-3^{\circ}\end{array}$ \\
\hline DCD & 117 & 0.4 & 0.83 & 2.48 & $\begin{array}{c}1.6 \\
\text { (at } 6 \mathrm{~m} \text { ) }\end{array}$ & 0.56 & $\begin{array}{c}2.68 \\
(1.3 \mathrm{GHz})\end{array}$ & $\begin{array}{c}0.8 \\
(1.3 \mathrm{GHz})\end{array}$ \\
\hline HIRA & 77 & 0.19 & 0.96 & 2.88 & $\begin{array}{c}7.5 \\
\text { (at } 6 \mathrm{~m} \text { ) }\end{array}$ & 2.65 & $\begin{array}{c}8.8 \\
(1.3 \mathrm{GHz}) \\
5.7 \\
(2.0 \mathrm{GHz})\end{array}$ & $\begin{array}{c}5.0 \\
(1.3 \mathrm{GHz}) \\
4.2 \\
(2.0 \mathrm{GHz})\end{array}$ \\
\hline SLH & 55 & 0.05 & - & - & - & $\sim 1$ & $\begin{array}{c}14.5 \\
(1.3 \mathrm{GHz}) \\
28.1 \\
(2.0 \mathrm{GHz})\end{array}$ & $\begin{array}{c}1.7 \\
(1.3 \mathrm{GHz}) \\
0.8 \\
(2.0 \mathrm{GHz})\end{array}$ \\
\hline
\end{tabular}

substantially different in amplitude (Figs. 5(c) and 5(d)). When the amplitude (power) of oscillations at the NLTL output is $U_{\mathrm{OSC}}=17 \mathrm{kV}$ (i.e., $P_{\mathrm{OSC}} \approx 3 \mathrm{MW}$ ) the $\mathrm{DCD}$ emitter (oriented in an optimal way with respect to the $\mathrm{SLH})$ provides signal levels in the receiving antenna like $P_{\mathrm{R}} \approx 1.6 \mathrm{~kW}$ (specifically, the amplitude of the first voltage surge is $U_{\mathrm{R}}=0.4 \mathrm{kV}$ ). In the case of the HIRA (also of optimal orientation toward the SLH) the estimate for the received amplitude (power) is as high as $U_{\mathrm{R}}=1.1$ $\mathrm{kV}\left(P_{\mathrm{R}} \approx 12 \mathrm{~kW}\right)$. The spectral width of the oscillations received (Fig. 5(a)) is close to the widths of the radiated spectra (Fig. 3(b) and Fig. 4(b)). The last column of Table 2 presents gain factors for the three antennas (at $f_{0}=1.3$ $\mathrm{GHz}$ and $f_{0}=2 \mathrm{GHz}$ ) as calculated for the conditions of a real measuring session where the antennas might not be optimally oriented toward one another but rather pointed 'generally along the horizontal' near the ground plane (within the range of elevation angles $\vartheta=0-3^{\circ}$ ).

\section{Results of Experiments with a NLTL Matched to WB Radiating Antennas}

In the radiation experiments, the whole system consisting of the high-voltage pulse generator, transmission lines and antennas was disposed vertically. Thus, the TX antennas were at a height of $2 \mathrm{~m}$, and the receive antennas could be placed in the far region with respect to the emitters, specifically at a distance $L=6 \mathrm{~m}$ (indoors) or at $L=20 \mathrm{~m}$ (outdoors). In the room, the RX antennas stayed at the same height above the floor as the DCD or the HIRA. Unfortunately, it was not always possible to provide for an optimal orientation of the antennas with respect to each other and/or other objects in the premises. The emitted signal was received either by the SLH antenna $(f=0.4-4 \mathrm{GHz})$ or by a standard WB horn antenna P6-23A (operating range $f=0.8-12 \mathrm{GHz}$ ). The signals were recorded with an Agilent 54846A digital oscilloscope (bandwidth $2.25 \mathrm{GHz}$ ), as voltages at the 50 $\Omega$ output loads of the receive antennas. Shown in Fig. 6 are examples of the received signals and of their spectra, obtained for typical transmitting conditions (i.e. peak voltage $U_{0}$ of the initial driving pulse and magnitude of the bias field $H_{0}$ which control the generation frequency). The relative radiation efficiency of the two transmitting antennas can be estimated either from the real-life or numerical experiments by comparing the magnitudes of their respective receive-transmit ratios,

$$
K=P_{\mathrm{R}} / P_{\mathrm{RAD}},
$$

where $P_{\mathrm{R}}$ is the RF power measured in the receive antenna and $P_{\mathrm{RAD}}$ the radiated power. If these two magnitudes are taken for a specific frequency $f$ (i.e., understood as parameters of a quasi-monochromatic RF pulse), then the ratio $K$ can be expressed as

$$
K(f)=P_{\mathrm{R}} / P_{\mathrm{RAD}}=G_{1}(f) \cdot G_{2}(f) c^{2} /(4 \pi)^{2} f^{2} L^{2} .
$$

Here $G_{1}(f)$ and $G_{2}(f)$ are directive gains of the transmit and the receive antenna, respectively, which are to be taken for the actual geometric configuration of each measuring session as proper readings from the antenna pattern graphs of Figs. 3(d); 4(d), and 5(c) (also see the last column of Table 2). The parameter measured in the experiment is the voltage $U_{\mathrm{R}}$ across the matched load $Z_{\mathrm{A}}$ of the receive antenna, whence the received power $P_{\mathrm{R}}$ is

$$
P_{\mathrm{R}}=1 / 2 U_{\mathrm{R}}^{2} / Z_{\mathrm{A}}
$$

The values of $P_{\mathrm{R}}$ obtained from measurements with all transmit-receive antennas pairs are listed in column 3 of Table 3. Taking the values of the TX antenna gains $G_{1,2}(f)$ pertaining to each geometric configuration of the 
DCD-to-SLH $U_{0}=88 \mathrm{kV} ; U_{\mathrm{OSC}}=17 \mathrm{kV} ; H_{0}=47 \mathrm{kA} / \mathrm{m}$
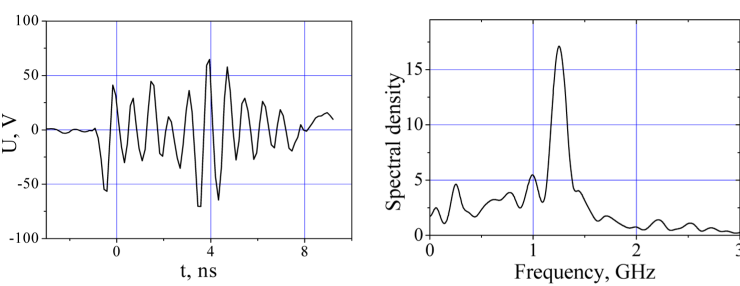

HIRA-to-SLH

$U_{0}=140 \mathrm{kV} ; U_{\mathrm{OSC}}=17 \mathrm{kV} ; H_{0}=53 \mathrm{kA} / \mathrm{m}$
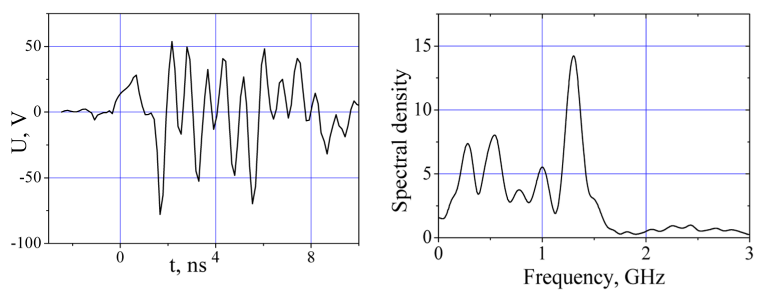

DCD-to-SLH

$U_{0}=178 \mathrm{kV} ; U_{\mathrm{OSC}}=12 \mathrm{kV} ; H_{0}=88 \mathrm{kA} / \mathrm{m}$

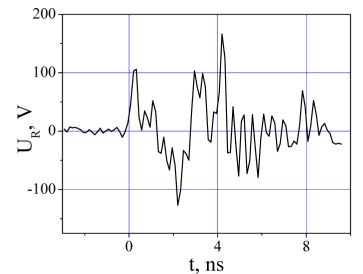

HIRA-to-WB Standard horn $U_{0}=112 \mathrm{kV} ; U_{\mathrm{OSC}}=17 \mathrm{kV} ; H_{0}=53 \mathrm{kA} / \mathrm{m}$
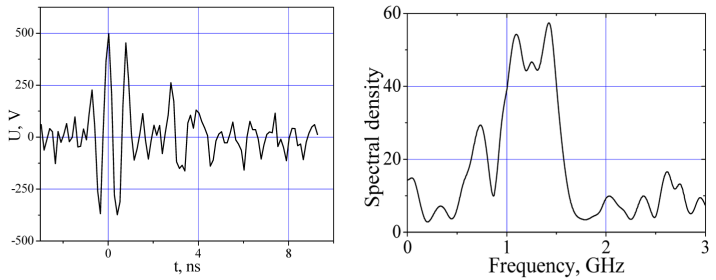

DCD-to-SLH

$U_{0}=120 \mathrm{kV} ; U_{\mathrm{OSC}}=12 \mathrm{kV} ; H_{0}=52 \mathrm{kA} / \mathrm{m}$
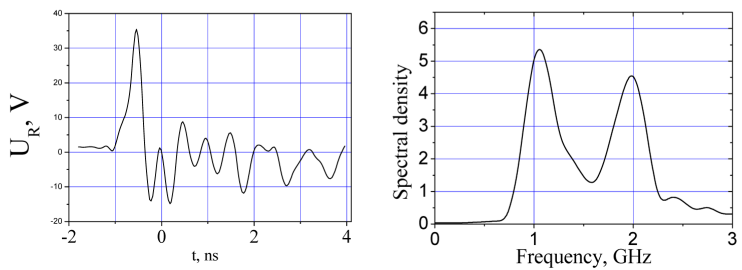

Fig. 6. (Color online) Signals received by the strip-line horn and the standard horn antenna under various experimental conditions, and the related spectra. The output characteristics corresponding to the plots are listed in Table 3.

Table 3. Radiated power levels from the DCD and the HIRA, as calculated for several experimental conditions.

\begin{tabular}{|c|c|c|c|c|}
\hline Antennas & $\begin{array}{l}\text { Oscillating power } \\
P_{\mathrm{OSC}}, \mathrm{W} \\
\end{array}$ & $\begin{array}{l}\text { Received power, } \\
\quad P_{\mathrm{R}}, \mathrm{W} \\
\end{array}$ & $\begin{array}{c}\text { Radiated power } \\
P_{\mathrm{RAD}}, \mathrm{W}\end{array}$ & $\begin{array}{c}\text { Radiation efficiency, } \\
\text { R }\end{array}$ \\
\hline $\begin{array}{l}\text { DCD-to-SLH } \\
f=1.3 \mathrm{GHz}\end{array}$ & $3 \times 10^{6}$ & $\begin{array}{c}(L=6 \mathrm{~m}) \\
25\end{array}$ & $1.9 \times 10^{6}$ & 0.63 \\
\hline $\begin{array}{l}\text { DCD-SLH } \\
f=2.2 \mathrm{GHz}\end{array}$ & $1.44 \times 10^{6}$ & $\begin{array}{c}(L=6 \mathrm{~m}) \\
16\end{array}$ & $1.1 \times 10^{6}$ & 0.76 \\
\hline $\begin{array}{l}\text { HIRA-to-SLH } \\
f=1.3 \mathrm{GHz}\end{array}$ & $3 \times 10^{6}$ & $\begin{array}{c}(L=8 \mathrm{~m}) \\
49\end{array}$ & $2.4 \times 10^{6}$ & 0.8 \\
\hline $\begin{array}{l}\text { HIRA-to-WB Standard } \\
\quad f=1.3 \mathrm{GHz}\end{array}$ & $3 \times 10^{6}$ & $\begin{array}{c}(L=5 \mathrm{~m}) \\
2500\end{array}$ & $2.4 \times 10^{6}$ & 0.8 \\
\hline $\begin{array}{c}\text { Outdoors site, DCD-to-SLH } \\
\qquad f=2 \mathrm{GHz}\end{array}$ & $1.44 \times 10^{6}$ & $\begin{array}{c}(L=20 \mathrm{~m}) \\
6.3\end{array}$ & $0.9 \times 10^{6}$ & 0.63 \\
\hline
\end{tabular}

experiment we are in a position to estimate the radiated power from Eq. (4) as $P_{\mathrm{RAD}}=P_{\mathrm{R}} / K$. Finally, we can estimate the radiation efficiencies of the transmitting antennas, $R=P_{\mathrm{RAD}} / P_{\mathrm{OSC}}$, for several cases. The results are presented in Table 3. As can be seen, both transmitting antennas demonstrate acceptable levels of the 'monochromatic' radiation efficiency. The HIRA-type antenna definitely seems more efficient owing to its better focusing ability compared with the DCD (recall that the azimuthal- plane pattern of the DCD is practically uniform). Experimental estimates for absolute values of the radiated RF power are somewhat lower than the magnitudes obtained numerically, however demonstrating a better agreement than just an order of magnitude coincidence. The obvious reason for these discrepancies is the difficulty of providing a proper electromagnetic environment for the experiment.

Apart from the radiation-focused aspect of this work, 

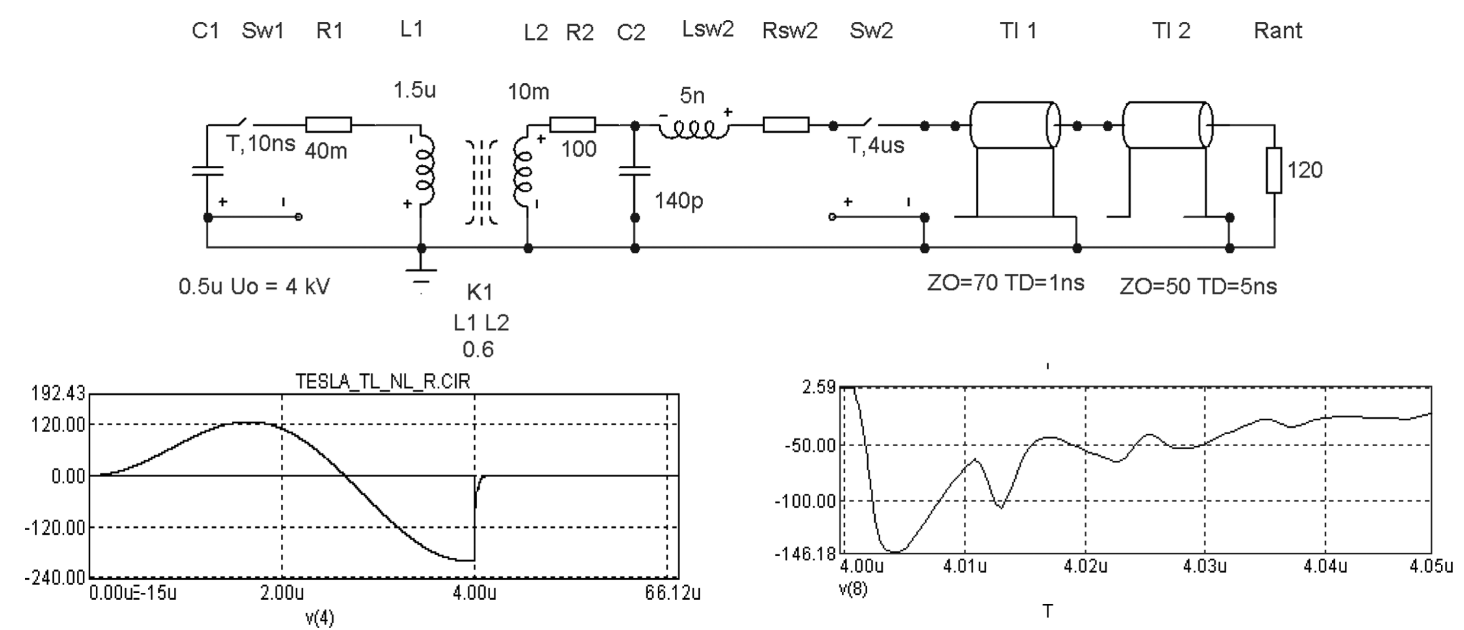

Fig. 7. Simplified equivalent circuit of a pulsed high-voltage source to generate the 'composite' signal with reflections. The linear TL2 is a substitute for the real NLTL of impedance $\mathrm{Z}_{\mathrm{TL} 2}=\mathrm{Z}_{\mathrm{NLsat}} \approx 50 \Omega$ corresponding to its saturated state.

we would like to note an interesting feature of the RF pulses that may be useful for the formation of 'combined' signals in the NLTL. As can be seen in Fig. 6, the waveform of the received signal is, more often than not, complicated by the presence of two bursts which are separated by 4 to $6 \mathrm{~ns}$ and each followed by oscillating components. The reasons for the appearance of this combined signal become clear if we consider that initially the dc pulse traveling from the high-voltage impulse source into the coaxial transmission system comes across a non-matched NLTL load. The mismatched signal that appears returns into the Tesla transformer and then goes back into the NLTL. The separation between these pulses equals the double travel time through the short transmission line TL1 (Fig. 7). The second (reflected) impulse travels along the NLTL in an already "prepared" ferrite environment and causes additional excitation of the NLTL. It seems interesting to note that in some cases the second signal excites oscillations of a higher intensity than the one running in front of it.

This experimental fact was investigated by means of electric-circuit simulation based on the Micro-Cap 7.0 software. For simplicity, the NLTL can be replaced by a linear TL2 of an impedance $Z_{T L 2}$ corresponding to the saturated state of the NLTL $\left(Z_{\mathrm{NLsat}} \approx 50 \Omega\right)$. As can be seen from the plots, due to the reflections occurring between TL1 and the capacitor $C 2$ in the secondary circuit of the Tesla transformer the transmission line TL2 receives the signal V8 which contains a depression in its decaying part. Respectively, an additional surge signal of a magnitude depending upon the mismatch between the lines TL1 and TL2 appears at the antenna terminals (across the resistor $R_{\mathrm{ANT}}$ ). In the real circuit containing the
NLTL this surge signal gives rise to repeated oscillations, which are actually observed in the experiment.

\section{Conclusions}

Two types of antennas have been analyzed numerically and experimentally, which can be used for radiating the quasi-harmonic wideband signals generated in a nonlinear transmission line with a pre-magnetized ferrite medium, subject to forcing by a pulsed current. The results for the power radiated from the disc-cone dipole (DCD) and the HIRA-type antenna, calculated for different experimental configurations and frequencies, allow us to draw the following, designs of both antennas are easily adaptable to the coaxial feeder; lack of intermediate elements (serving to convert the TEM wave of a coaxial feeder into a TE mode of a circular horn antenna) allows simplifying the design of the output unit of the NLTL-based UWB radiating system; active low-inductance resistors allow for good matching between the antenna and the source of the complex WB signal in the lower frequency range (up to 0.3-0.4 GHz), while the geometry of the antenna electrodes allows simple matching of these units in the upper frequency range $(0.5-3 \mathrm{GHz})$; the DCD antenna demonstrates a significantly lower radiation efficiency for the combined impulse signal in comparison with the HIRA, which effect can be explained by difficulties in matching the antenna and the feedline over the whole range of emitted frequencies; thus, the radiation efficiency of the DCD antenna does not exceed $60 \%$ for $f_{0}=1.3 \mathrm{GHz}$ and $70 \%$ for $f_{0}=2.2 \mathrm{GHz}$, while for the HIRA this parameter is close to $90 \%$. 


\section{References}

[1] A. M. Belyantsev, Y. K. Bogatyryov, and L. I. Solovyova, Izv. VUZ-Radiofizika 6, 551 (1963).

[2] J. A. Gaudet, E. Schamiloglu, J. O. Rossi, C. J. Buchenauer, and C. Frost, In: Proc. of the 2008 IEEE Int'l 'Power Modulators and HV' Conference, Las Vegas, NV, USA, 131 (May 2008).

[3] C. R. Spikings, N. Seddom, and J. E. Dolan, IET Conference on High Power RF Technologies, 1 (2009).

[4] V. V. Rostov, N. M. Bykov, B. N. Bykov, A. I. Klimov, O. B. Kovalchuck, and I. V. Romanchenko, IEEE Trans. Plasma Sci. 38, 2681 (2010).

[5] I. V. Romanchenko, V. V. Rostov, V. P. Gubanov, A. S. Stepchenko, A. V. Gunin, and I. K. Kurkan, Rev. Sci. Instrum. 83, 074705 (2012), doi: 10.1063/1.4738641.

[6] J.-W. Ahn, F. O. Dryakhlov, S. Yu. Karelin, V. B. Kra- sovitsky, H.-O. Kwon, I. I. Magda, A. A. Markova, V. S. Mukhin, and B. G. Zagvozdkin, 25th Int'l Conf. on Microwave and Telecomm. Technology, Sevastopol, Crimea, 785 (2015).

[7] J.-W. Ahn, S. Y. Karelin, H.-O. Kwon, I. I. Magda, and V. G. Sinitsin, J. Magn. 20, 460 (2015).

[8] S. A. Berdin, N. P. Gadetski, E. I. Kravtsova, J. Lee, I. I. Magda, V. S. Mukhin, J. H. Ryu, V. G. Si-nitsin, and I. A. Vyazmitinov, IEEE Trans. Plasma Sci. 41, 2539 (2013).

[9] S. A. Schelkunoff and H. A. Friis, Antennas: Theory and practice, Wiley, New York (1952).

[10] E. G. Farr, C. E. Baum, and C. J. Buchenauer, in: L. Carin, L. Felsen (Eds.), Ultra Wideband/Short-Pulse Electromagnetics 2, Plenum Press, New York (1995), pp. 159-178.

[11] https://www.cst.com/Products/CST MWS. 